\title{
Ether Island: Investigating sound movement correlations in a generative interactive installation
}

\author{
Andrey Bundin \\ Herzen State Pedagogical University \\ St. Petersburg, \\ Russia \\ ibundin@gmail.com
}

\author{
Natalia Fedorova \\ St Petersburg State University \\ St Petersburg, \\ Russia \\ phd.natali@gmail.com
}

\begin{abstract}
Ether Island is an interactive sound installation that creates sonic spaces of the sounds human ear cannot normally register if unarmed: solar wind, satellite signal, communication between two radios on air; and invites visitors to make gestures with their hands to explore relations between sound and movement. According to Konstantin Tsiolkovsky ether island - is all the known universe in present future and past. Software gets skeleton data of a visitor and transmits it to sound engine, which generates speech sounds and noises depending on the position of visitor's hands. It is achieved by using infrared body tracking sensors (currently Microsoft Kinect) along with sound synthesis and processing algorithms implemented in Max.
\end{abstract}

interactive sound installation. touchless instrument. Russian cosmism.

\section{INTRODUCTION}

The paper describes ideological and technical principles of Ether Island created by Andrey Bundin and Natalia Fedorova for an exhibition dedicated to the media archeological research of the past of radio as a medium and its possible meanings today. Private Life of Radio Frequencies opened on 7 May 2018 at the Museum of Telecommunication in St Petersburg, Russia. The aim of the exhibition was to give air to the inaudible and the unheard including atmosphere, radio pulsars, no longer used long waves, recordings of the air of the radio stations of the past etc.

During the Private Life of Radio Frequencies Ether Island was mounted at the feet of Lutch - 15, a geostationary artificial Earth satellite registered with an international index SDRN (W,C,E). Lutch -15 was projected for telebridges and real-time broadcasting, but was never launched. The visual landmark of the First Atrium of the Museum, Lutch-15 hosted two installations. The second one, Radiopulsar was a collaboration of an artist Alexey Khamkin and radio astronomer Andrey Kazantsev, mounted on the solar batteries. The artwork visualized the white noise of dynamic spectors electromagnetic radiation of radiopulsars, fast moving neutron stars.

Ether Island comprised two distinct sound streams associated with the right and the left hand. The right hand track evokes the voices that were part of the ether of the past: communication between Kedr 1

(Yury Gagarin, the first cosmonaut) and Zaria 1 (father founder of rocket engineering Sergey Korolev). Despite its historic meaning this recorded communication was top secret and has never been broadcast on the radio. Here radio operates not as a transmissional media, but as a communication device. The installation in this case recreates a possible ether of the alternative past. The transmission-broadcasting dichotomy of the earth-orbit communication was historically gravitating towards closed channels of transmission only disregarding the broadcasting for the reason that even scientific part of the Soviet space program was in service of its military component and thus has always been top secret.

The second track that is used in the right hand stream The War of the Worlds by Herbert Wells, an episode of the American radio drama series The Mercury Theatre on Air. It was directed and narrated by Orson Welles on Sunday, October 30, 1938. Unaccustomed to radio plays American public partially took the news broadcast of Martian invasion of earth for real which caused mass panic. Here radio was used as a broadcasting media, reporting alternative reality of the ether island.

Left hand of the visitor controls volume and spectral quality of several noises. Horizontal movements crossfade between different radio noises received from space: solar wind, cosmic background noise, and the noises emitted by different planets Those recordings were converted to sound files with audible spectral and amplitude range. Vertical movement of a visitor's left hand controls the quality of sound: it is mapped to cutoff frequency and resonance level of the low-pass filter applied to the track, and to the amount of sound effects added (distortion and flanger). 
Together all the left hand noises created complex sonic image of galactic ether. The right hand voices were translating talking away and talking out of the fear of death to the universe into a physical experience.

Even though these sonic environments are capable of translating both the physical sensation of ether as a physical and thought space the complexity of the concept itself requires further elaboration. Within the scope of cosmist ideas ether not only included all the known universe in present, but also all of its inhabitants from the past. According to Nikolay Fedorov's ideas in the Philosophy of Common Good all ever living humans need to be resurrected as works of art. This is the only possibility for building a state based on justice, because if otherwise the ones who were contributing to better life never get a chance to live in it. Collection of resurrected fathers should form the museum in its most correct function, museum as a collection of all the people never to be mortal again. Thus further expansion of the Ether Island is needed to include this perspective of time.

\section{FURTHER DEVELOPMENT OF THE WORK: ETHER ISLAND IN VR}

In the current version of the installation, we use infrared body tracking sensors (Microsoft Kinect) isolating small groups of people with limited recourses for extended periods of time: BIOS, Krasnoyarsk, USSR, 1972, Biosphere-2, 1991-1993, ISEMSI, Bergen, 1990, SFINCSS-99,Russia, 1999-2000, Mars 500, Russia, 2010-2011, HI-SEAS,US, 2015-now). The universe part will be worded with the texts of Russian cosmists (Nikolay Fedorov, Philosophy of Common Good, Konstantin Tsiolkovsky, Ether Island and others) and fiction texts dedicated to interplanetary travelling of humans and non-humans (Herbert Wells, War of the Worlds). By moving in the this virtual space, an observer travels in time and space of radio ether.

Every virtual station is mapped to particular position in a sphere of Ether Island. Waves (recordings of radio broadcasts, radio communication) are emitted from the central point of the virtual sphere and spread in multiple directions to be reflected form the edges of the sphere and then travel at a random direction. Simultaneously the sound field of every wave is growing and at a certain moment the visitor, who is initially at almost soundless space, cannot escape from constantly circulating sounds. This lasts until their fields don't coincide with the field of the universe and the ether is not filled with almost white noise. Then the visitor hears all the sounds simultaneously in every point. This stands for the collapse of The Ether Island, followed by momentary silence. And the rays of waves continue circulating from the beginning again. and sound synthesis and processing algorithms implemented in Max. Software gets skeleton data of a visitor and transmits it to sound engine, which generates different sounds depending on the position of visitor's hands. It creates strong mind feedback loop: brain - gesture - sound - brain.

In the next stage we plan to port the installation in VR using Unity. The key concept of the project bases on the consideration of virtual environment as an equality of Tsiolkovskian Ether island, all known universe, but also a part of an Ether ocean. Ether ocean is an agglomeration of Ether islands moving in an unknown direction at a speed comparable to unknown universes. The word ether in terminology of Konstantin Tsiolkovsky means the atmosphere. In our work we use ether both as an atmosphere and wireless space and time for radio broadcasting.

Emotionally Ether Island translates to the experience of a human in a closed space or sensory deprivation camera juxtaposed against giant universe. The source text used will be sound space experimental recordings (Alvin Lucier I am sitting in the room) and documentary materials form the known space simulating experiments such as a book Earth A Year in the Earth Space Craft by Andrey Bozhko and Violetta Gorodinskaya. Other international space research preparation projects that took place on earth may include and involved

\section{Technical realization of Ether Island in VR project}

Installation dynamically computes the distances (D) between observer's personage location and all virtual stations using simple Euclidean space principals:

Next, using this data, it calculates volume levels for

$$
D_{n}=\sqrt{\left(L_{x n}-S_{x n}\right)^{2}+\left(L_{y n}-S_{y n}\right)^{2}+\left(L_{z n}-S_{z n}\right)^{2}}
$$

all virtual radio waves:

$$
V_{n}=\max \left(0, \min \left(1,1-D_{n} / W_{n}\right)\right)
$$

Where $\mathrm{n}$ is the number of virtual radio stations, $\mathrm{V}$ is the volume of certain station and $W$ is its weight. In this function weight is the radius of a spherical field in which an observer can hear particular station or several stations with different volumes simultaneously, the closer the listener to a station the louder the sound it produces.

Spherical sound field of each radio wave grows from the moment it was produced and until it becomes equal to the sphere of the virtual universe:

$$
W_{n}=\left(t-t_{n}\right) /\left(t_{\max }-t_{n}\right) * R
$$

Where tis current time, is the time when a certain 
radio wave has been emitted, $t_{\max }$ is the moment in future when Ether Island will collapse, and $R$ is the radius of the whole spherical virtual space (the universe).

One of the problems of the work is to find proper sounds to create the artistic interpretation of Tsoilkovskian Ether island. During the development stage, we found two approaches: using real galactic sounds and documental recordings of radio broadcasts of the past and creating emotional feedback by using musical elements, specifically designed sound, and storytelling. And because our work doesn't have to be documentary, we focused on the second approach.

Empty areas between virtual radio waves are filled with quite monotone low-frequency noises with floating low-pass resonant filters creating the effect of blowing wind. Psychoacoustic study, dedicated to the impact of low-frequency noise and sound quality evaluation, reveals that the sounds of this kind create emotional feedback. The artistic aim of using quite low-frequency filtered noise is to make the listener feel sad and lonely in the eternal space. One of the artistic problems of the work is to decide what kind of sounds can create this emotional feedback

\section{Conclusion}

Paper presented both the ad hoc condition of the work and perspective for its future development. Present generative composition has been demonstrated at the Museum of Telecommunication as an installation as well as at the National Centre for Contemporary Art as a performance piece. Both these demonstrations revealed that the intricacy of the concept of ether demands for not only sonic interpretation of physical and thought spaces, but also the space of time. This justifies looking into VR as a possibility for this third dimension. As the Island itself in Tsiolkovskian concept is a unity in the state of flux assembling, developing and eventually collapsing the same logics will be implemented to the movement of the sound waves. Waves emitted from the central point of the virtual sphere and spread in multiple directions reflect form the edges. of the sphere and then travel at a random directions. This will allow for the sound field of every wave to be growing and create inescapable circulation of sounds until their fields coincides with the field of ether itself and filled with almost white noise. It will make visitor hear all the sounds simultaneously in every point and stand for the collapse of the Ether Island, followed by momentary silence. And the rays of waves continue circulating from the beginning again. Use of VR will not be a mere translation of time dimension, but rather a possibility of translation of the idea of complex movement of an Ether island within the Ether ocean.

\section{REFERENCES}

Fedorov, N (1982). Sochineniya.Filosofskoye naslediye. Mysl', Moscow

Gerovich, S. (2015). Soviet Space Mythologies. Public Images, Private Memories, And The Making of Cultural Identity. University of Pittsburgh Press

Tsiolkovsky, K. (2013) Ephyrny ostrov. O०० "Tsentr informatsionnoy bezopasnosty", http:// tsiolkovsky.org/wpcontent/uploads/2016/02/Efirnyj-ostrov.pdf 